\title{
Minimum Electric Field Exposure for Seizure Induction with Electroconvulsive Therapy and Magnetic Seizure Therapy
}

\author{
Won H Lee', Sarah H Lisanby, ${ }^{2,3,7}$, Andrew F Laine ${ }^{4}$ and Angel V Peterchev ${ }^{*, 2,5,6}$ \\ 'Department of Psychiatry, Icahn School of Medicine at Mount Sinai, New York, NY, USA; ${ }^{2}$ Department of Psychiatry and Behavioral Sciences, \\ Duke University, Durham, NC, USA; ${ }^{3}$ Department of Psychology and Neuroscience, Duke University, Durham, NC, USA; ${ }^{4}$ Department of \\ Biomedical Engineering, Columbia University, New York, NY, USA; ${ }^{5}$ Department of Biomedical Engineering, Duke University, Durham, NC, USA; \\ ${ }^{6}$ Department of Electrical and Computer Engineering, Duke University, Durham, NC, USA
}

Lowering and individualizing the current amplitude in electroconvulsive therapy (ECT) has been proposed as a means to produce stimulation closer to the neural activation threshold and more focal seizure induction, which could potentially reduce cognitive side effects. However, the effect of current amplitude on the electric field (E-field) in the brain has not been previously linked to the current amplitude threshold for seizure induction. We coupled MRI-based E-field models with amplitude titrations of motor threshold (MT) and seizure threshold (ST) in four nonhuman primates (NHPs) to determine the strength, distribution, and focality of stimulation in the brain for four ECT electrode configurations (bilateral, bifrontal, right-unilateral, and frontomedial) and magnetic seizure therapy (MST) with cap coil on vertex. At the amplitude-titrated ST, the stimulated brain subvolume (23-63\%) was significantly less than for conventional ECT with high, fixed current (94-99\%). The focality of amplitude-titrated right-unilateral ECT (25\%) was comparable to cap coil MST (23\%), demonstrating that ECT with a low current amplitude and focal electrode placement can induce seizures with E-field as focal as MST, although these electrode and coil configurations affect differently specific brain regions. Individualizing the current amplitude reduced interindividual variation in the stimulation focality by $40-53 \%$ for ECT and $26 \%$ for MST, supporting amplitude individualization as a means of dosing especially for ECT. There was an overall significant correlation between the measured amplitude-titrated ST and the prediction of the E-field models, supporting a potential role of these models in dosing of ECT and MST. These findings may guide the development of seizure therapy dosing paradigms with improved risk/benefit ratio.

Neuropsychopharmacology (2017) 42, I192-1200; doi: I0.1038/npp.2016.276; published online 25 January 2017

\section{INTRODUCTION}

Electroconvulsive therapy (ECT) remains the fastest acting and most effective established treatment for severe major depression (Kellner et al, 2010; Weiner, 2015), including a rapid relief of suicidal intent (Kellner et al, 2005). Both its efficacy and adverse side effects depend on the electrical stimulation parameters (Peterchev et al, 2010; Rasmussen, 2015). Consequently, various ECT technique modifications have been proposed and developed to reduce cognitive side effects by making the electric stimulus closer to the neural activation threshold and more focal. For example, high-dose right unilateral (RUL) ECT has comparable efficacy to

* Correspondence: Dr AV Peterchev, Department of Psychiatry and Behavioral Sciences, Duke University, Box 3620 DUMC, Durham, NC 277I0, USA, Tel: 919684 0383, Fax: 9196819962 ,

E-mail: angel.peterchev@duke.edu

${ }^{7}$ Now at the National Institute of Mental Health. Dr Sarah H Lisanby contributed to this article while at Columbia University and Duke University, prior to joining NIMH. The views expressed are her own (the authors) and do not necessarily represent the views of the National Institutes of Health or the United States Government.

Received 19 May 2016; revised 5 November 2016; accepted 4 December 2016; accepted article preview online 9 December 2016 bilateral frontotemporal (BL) ECT with a significant decrease in amnesia (Sackeim et al, 2000; Semkovska et al, 2016). Alternative electrode placements that are more focal than BL also include bifrontal (BF) and an experimental frontomedial (FM) configuration, which target the electric field (E-field) in the prefrontal cortex (Kellner et al, 2010; Lee et al, 2016). Also, reducing the pulse width decreases the stimulation strength and increases focality, resulting in reduced cognitive side effects (Tor et al, 2015). Finally, magnetic seizure therapy (MST) induces a significantly weaker and more focal E-field than conventional ECT, which appears to diminish cognitive side effects (Cretaz et al, 2015; Kayser et al, 2015; Lee et al, 2016). To be effective, stimulation with briefer, more focal E-field pulses appears to require a larger number of pulses (Loo et al, 2014; Sackeim et al, 2000). Thus understanding the relationship between the stimulus parameters, the strength and focality of the induced E-field, and the resultant seizure can inform clinical studies seeking to optimize ECT and MST.

The stimulus current amplitude is a key factor controlling the stimulation strength and focality but has received little attention in modern ECT studies. We recently explored the minimum individual current amplitude required to induce seizures with several ECT electrode placements and with 
MST in nonhuman primates (NHPs) (Peterchev et al, 2015). That study found the amplitude-titrated seizure threshold (ST) to be 4-8 times lower than conventional ECT current amplitude and to vary significantly across subjects. However, the reduction of the current amplitude required for seizure induction is expected to result in part from the smaller head size of the NHPs compared with humans, confounding the relevance to clinical procedures. Also, the relationship between the amplitude of the current applied to the ECT electrodes or MST coil on the scalp and the strength, distribution, and focality of the E-field induced in the brain is unknown, limiting understanding of the specific brain areas stimulated by the various paradigms. Finally, it is unclear to what extent individual differences in the amplitude-titrated ST are driven by anatomical variation that can be quantified and used for dose selection.

Addressing these questions, in this paper we investigate the E-field characteristics of ECT and MST with individualized current amplitude using magnetic resonance imaging (MRI)-based anatomically realistic computational head models in a subsample of four NHP subjects of our prior study (Peterchev et al, 2015). The neural activation threshold is approximated from the measured individual motor threshold (MT) and the simulated E-field strength. This allows estimation of the stimulation strength and focality at the individual amplitude-titrated ST and contrasting them to those for fixed, non-individualized current amplitude. Further, we explore how the focality of stimulation is affected by the current amplitude as well as by the ECT electrode and MST coil configuration. Considering the E-field characteristics in addition to the stimulus parameters allows for the first time a direct, quantitative comparison with ECT and MST in humans. Understanding the induced E-field characteristics across electrode/coil configurations, current amplitudes, and individuals could help identify potential causes of differences in clinical outcomes and could support the development of dosing paradigms with fewer side effects.

\section{MATERIALS AND METHODS}

\section{Subjects}

The subjects were four healthy male rhesus macaques (Macaca mulatta; age $=13-19$ years; weight $=8.4-10.7 \mathrm{~kg}$ ). All studies were performed using protocols approved by the Institutional Animal Care and Use Committees of New York State Psychiatric Institute, Columbia University, and Duke University.

\section{ECT and MST Model Generation and Simulation}

The construction of the head models of the four NHP subjects is described in detail previously (Lee et al, 2015). Briefly, the four subjects underwent T1-weighted structural MRI with $0.7 \times 0.7 \times 0.7 \mathrm{~mm}^{3}$ voxel size and diffusionweighted imaging with $1.4 \times 1.4 \times 1.4 \mathrm{~mm}^{3}$ voxel size. After image preprocessing, the tissue was segmented semiautomatically into gray matter, white matter, cerebrospinal fluid, skin, muscle, skull spongiosa, skull compacta, vertebrae, spinal cord, lens, eyeball, sclera, optic nerve, and sinus. All tissue compartments were assigned isotropic electrical conductivity from the literature, with the exception of white matter. The latter was assigned anisotropic conductivity tensors derived from the measured diffusion tensors and the isotropic white matter conductivity from the literature.

We modeled three standard ECT electrode placements (BL, BF, and RUL) (Kellner et al, 2010) and an investigational configuration (FM) (Rosa et al, 2011), as depicted in Figure 1 and described previously (Peterchev et al, 2015). For MST, we modeled a concave circular coil (CAP; MagVenture A/S, Farum, Denmark) consisting of a single-layer, concave circular winding with an inner diameter of $21 \mathrm{~mm}$, outer diameter of $95 \mathrm{~mm}$, and 15 turns. The coil conductors were centered above the vertex of the NHP head.

The complete rhesus macaque models incorporating the ECT electrodes or MST coil were adaptively meshed using the restricted Delaunay tessellation algorithm (Pons et al, 2007), resulting in 20 modality- and subject-specific finite

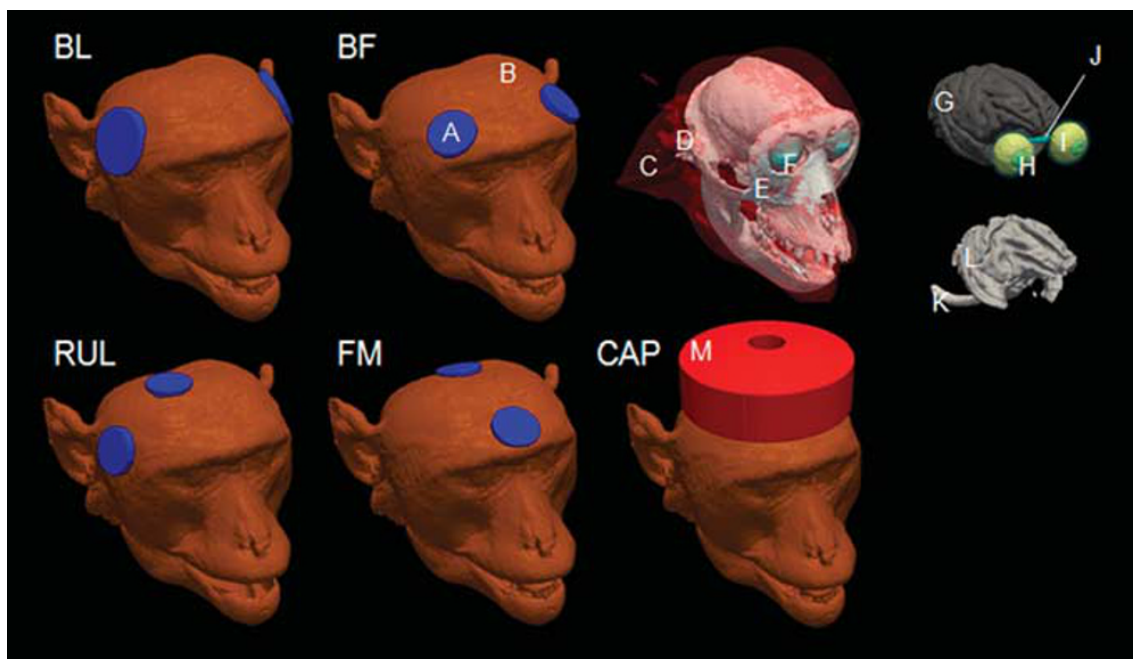

Figure I ECT and MST simulation models of a representative subject $(\mathrm{CH})$ for bilateral frontotemporal $(\mathrm{BL})$, bifrontal $(B F)$, right unilateral $(\mathrm{RUL})$, and frontomedial (FM) ECT as well as MST with cap coil centered on vertex (CAP). Labeled conductivity compartments are: (A) electrode, (B) skin, (C) muscle, (D) vertebrae, $(E)$ skull compacta, $(F)$ sclera, $(G)$ gray matter, $(H)$ lens, $(I)$ eyeball, $(J)$ optic nerve, $(K)$ spinal cord, $(L)$ white matter, and $(M)$ coil. 
element models. The E-field induced by ECT and MST in these models was simulated using the finite element method in ANSYS (ANSYS, Canonsburg, PA, USA), as described previously (Lee et al, 2015, 2016).

\section{In Vivo MTs and STs}

We previously reported amplitude-titrated MT and ST data for the four NHP subjects as part of a larger study (Peterchev et al, 2015). In summary, within the same session in each NHP we determined the MT corresponding to the amplitude of a single pulse required to elicit a motor response (under ketamine/xylazine sedation) and the ST corresponding to the amplitude for a train of pulses to elicit a seizure (under methohexital anesthesia and succinylcholine muscle relaxation). The ECT MT and ST were titrated by increasing the amplitude of stimulus pulses (pulse width $=0.2 \mathrm{~ms}$ ) delivered through the ECT electrodes with a DS7AH high-voltage constant-current stimulator (Digitimer, Welwyn Garden City, Hertfordshire, UK). The MST MT and ST were acquired using a MagPro MST device with the CAP coil (MagVenture A/S, Farum, Denmark). The coil induced in the head a cosine current pulse with clockwise initial phase direction and a $0.36 \mathrm{~ms}$ period. MST stimulus intensity is reported as percentage of maximum pulse amplitude (\% MA); maximum pulse amplitude corresponds to $1800 \mathrm{~V}$ peak coil voltage.

For MT, electromyography was measured from the first dorsal interosseous (FDI) muscle in both hands for all ECT and MST conditions but only from the left hand in the RUL ECT condition as RUL stimulation is predominantly unilateral in the right hemisphere. MT was defined as the minimum stimulus pulse amplitude needed to achieve a $50 \mu \mathrm{V}$ peak-to-peak motor evoked potential for at least 5 out of the 10 trials (Rossini et al, 1994). The ECT MT was determined for both current polarities. ST was obtained by an ascending method-of-limits titration of the stimulus pulse amplitude, while holding all the other stimulus parameters fixed. The ST titration steps were applied with an interval of at least $20 \mathrm{~s}$, consistent with clinical practice. The stimulus train consisted of 500 pulses delivered at 50 pulses per second resulting in train duration of $10 \mathrm{~s}$. We confirmed seizures by observing motor manifestations in the left arm and EEG as a secondary criterion. In each subject, the MT and ST titrations were repeated three times on 3 separate days.

\section{Data Analysis}

The neural activation threshold, $E_{\text {th }}$, was empirically estimated from the median E-field strength in the FDI representation of the motor cortex at stimulation current corresponding to the individual MT. For ECT, the RUL electrode configuration was used that produces the E-field distribution most localized to the FDI areas (Lee et al, 2015). For MST, the E-field thresholds were calculated for both left and right FDI regions and then averaged within subject.

We computed maps of the E-field strength relative to the threshold for neural activation by dividing the E-field magnitude distribution in the brain by the estimated threshold, $E / E_{\mathrm{th}}$. The focality of stimulation was quantified by the percentage of the brain volume that is exposed to E-field strong enough to produce suprathreshold depolarization in the majority of neurons, ie, the volume where $E / E_{\mathrm{th}} \geqslant 1$ (Deng et al, 2011). The stimulation strength and volume were modeled at the individual current amplitude corresponding to ST for each electrode and coil configuration in the four NHP subjects. The stimulated brain volume data were further analyzed with a mixed-effect model in JMP 10.0 (SAS Institute, Cary, NC, USA), with stimulation modality as a fixed effect and subject as a random effect. Significant results of the mixed-effect model were followed up with pairwise comparisons using Tukey's HSD test.

To explore further the differences in the spatial distribution of stimulation across the brain for the different ECT and MST modalities, we also characterized the E-field in three regions of interest (ROIs) in each hemisphere: primary motor cortex (FDI area), dorsolateral prefrontal cortex (DLPFC), and hippocampus. These ROIs are a subset of those analyzed previously in simulation studies of clinical ECT and MST (Lee et al, 2012a; Lee et al, 2016) and were selected owing to their putative involvement in seizure initiation, therapeutic efficacy for depression and suicidal ideation, and cognitive side effects (Kayser et al, 2015; Kellner et al, 2010; Reti, 2015; Sackeim, 2004; Sun et al, 2016), as well as owing to their representative locations and depths in the brain. The individual ROIs were determined based on the rhesus macaque brain stereotaxic atlases (Paxinos et al, 2000; Saleem and Logothetis, 2007) and the web-based Scalable Brain Atlas (http://scalablebrai natlas.incf.org).

To test whether individual differences in ECT/MST ST are predicted by individualized E-field computational models, we computed the ratio of the stimulation electrode or coil current, $I$, to the 90 percentile E-field strength in the brain, $E_{\text {brain }}$, for each ECT electrode and MST coil configuration. This ratio characterizes interindividual anatomical variation determined from individual E-field simulation models, which is expected to be correlated with individually titrated ST data. The 90 percentile E-field strength was selected for robustness and as at present it is unclear which brain regions are involved in seizure induction for each modality. To evaluate this relationship, we conducted a regression analysis of ST first within the four ECT modalities and then jointly for all ECT and MST modalities. In the latter, to make the measurement units compatible between ECT and MST, $I / E_{\text {brain }}$ and ST were normalized within each stimulation modality by calculating their $Z$-scores, which capture the individual variability within modality (Peterchev et al, 2015). In addition to normalized $I / E_{\text {brain }}$, session number was included as a factor. Other individual variables such as age and weight were not included as they did not significantly predict ST in the prior analysis of the data from this study (Peterchev et al, 2015). Subsequently, Pearson's linear correlation coefficient was computed for $I / E_{\text {brain }}$ and ST averaged across the three stimulation sessions within each modality. These correlations were of exploratory nature owing to the small number of subjects.

\section{RESULTS}

\section{Estimate of Neural Activation Threshold}

A representative individual model for E-field simulation of the ECT electrode configurations and the MST coil 

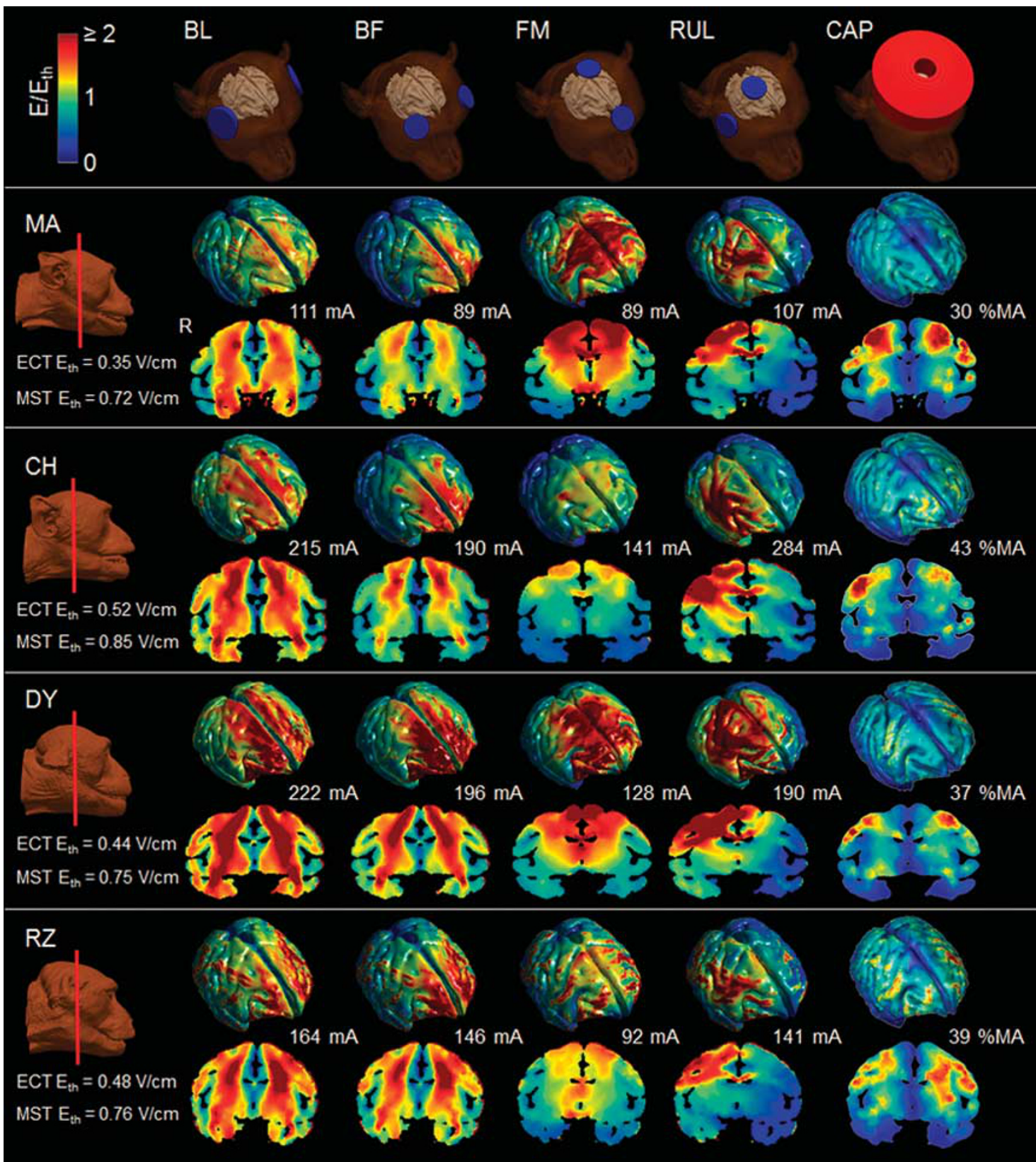

Figure 2 E-field strength $(E)$ relative to estimated neural activation threshold $\left(E_{\text {th }}\right)$ at current amplitude corresponding to amplitude-titrated ST for BL, BF, FM, and RUL ECT as well as CAP MST (second to fifth columns, respectively) for the models of subjects MA, CH, DY, and RZ. The stimulation strength E/E th is visualized on the cortical surface as well as in a representative coronal slice for each subject. The color map is clamped at an upper limit of 2 for good visibility. $R$, right.

configuration is displayed in Figure 1. The individual E-field simulations were first used to estimate the E-field threshold for neural activation, which was defined as the median E-field strength in the FDI motor area for stimulus current amplitudes equal to MT. MTs for RUL ECT and MST were $80 \pm 29 \mathrm{~mA}$ and $20 \pm 3 \% \mathrm{MA}$ (mean \pm SD), respectively. The corresponding estimated neural activation thresholds were $0.45 \pm 0.07$ and $0.77 \pm 0.06 \mathrm{~V} / \mathrm{cm}$ for RUL ECT and MST, respectively. The mean neural threshold was $71 \%$ higher for MST than for ECT $(t=-17.09, p<0.001)$, as expected because of the different pulse shape and duration (Deng et al, 2011).

\section{Spatial Distribution of Brain Stimulation at ST}

The estimated individual neural activation thresholds were used to normalize the individual E-field distributions to derive an approximate map of the stimulation strength across the brain. Figure 2 shows the resultant maps of stimulation strength in the brain for stimulus current amplitude equal to the individual amplitude-titrated ST. Figure 3 quantifies the stimulation strength in the three specific ROIs. The laterally symmetric configurations (BL, $\mathrm{BF}$, and FM ECT and CAP MST) induce similar stimulation patterns in both hemispheres with BL being most nonfocal, whereas the asymmetric RUL ECT electrode configuration 

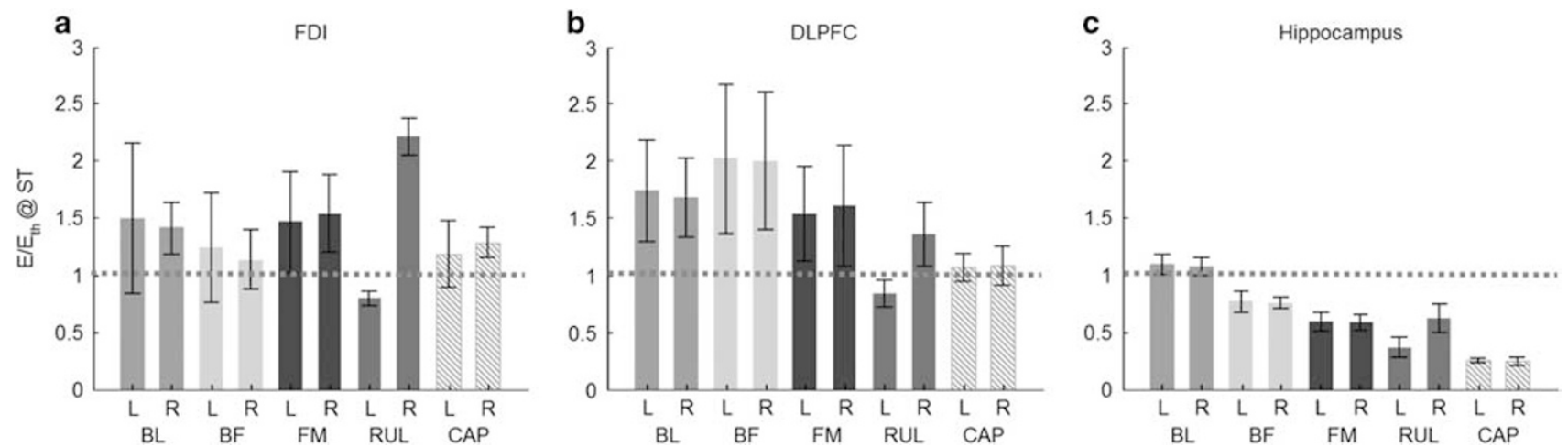

Figure 3 Stimulation strength $\left(E / E_{\text {th }}\right.$ ) at individual $S T$ in three brain ROls for BL, BF, FM, and RUL ECT as well as CAP MST. The ROls are (a) first dorsal interosseous (FDI), (b) dorsolateral prefrontal cortex (DLPFC), and (c) hippocampus. Bars correspond to the median stimulation strength within ROI in the left $(L)$ or right $(R)$ hemisphere averaged across the four subjects, and error bars give the SD across subjects. Horizontal gray dotted line demarcates the boundary between subthreshold and suprathreshold stimulation strength.

preferentially activates the right $v s$ the left hemisphere. The mostly right-sided suprathreshold stimulation by RUL ECT is in contrast to the bilateral stimulation spread when RUL ECT is delivered with conventional, high current amplitude (Lee et al, 2016). DLPFC is stimulated above the neural activation threshold for all modalities, although at different strengths, with BF ECT being the most intense. Compared with the ECT modalities, MST spares inferior regions bilaterally and stimulates hippocampi the least. Hippocampi are stimulated at suprathreshold level only by BL ECT with intensity approximately four times that of MST. Further, MST does not stimulate structures under the center of the circular coil on the vertex, whereas FM ECT strongly activates these regions. Indeed, FM ECT produces stronger deep medial stimulation than the other ECT configurations.

Quantitatively, there were significant differences in stimulated brain volume at ST across the five modalities (Figure 4a; $\mathrm{F}_{4,51}=45.9, p<0.0001$ ). The modalities formed three groups that were significantly different from each other $(p<0.02)$ : BL ECT had the highest stimulated volume $(63 \%)$, followed by BF (40\%) and FM (36\%) ECT, in turn followed by RUL ECT (25\%) and CAP MST (23\%). There was no significant effect on stimulated brain volume of subject or session number.

\section{Focality Control via Current Amplitude Adjustment}

Extending the results from Figure $4 \mathrm{a}$, Figure $4 \mathrm{~b}$ shows the simulated effect on focality of varying the current amplitude relative to the individual ST. As expected, the directly stimulated brain volume increases with higher current amplitude, but the shape of the curves relating them varies across the different modalities. For ECT, the curves are sigmoid and have a substantially higher maximum slope than the MST curve, which is more linear. The varying characteristics of the curves among the ECT modalities and MST are related to the different intrinsic focality characteristics of the electrode and coil configurations.

\section{Individualized vs Fixed Current Amplitude}

To evaluate the effectiveness of current amplitude individualization as a means to reduce stimulation strength
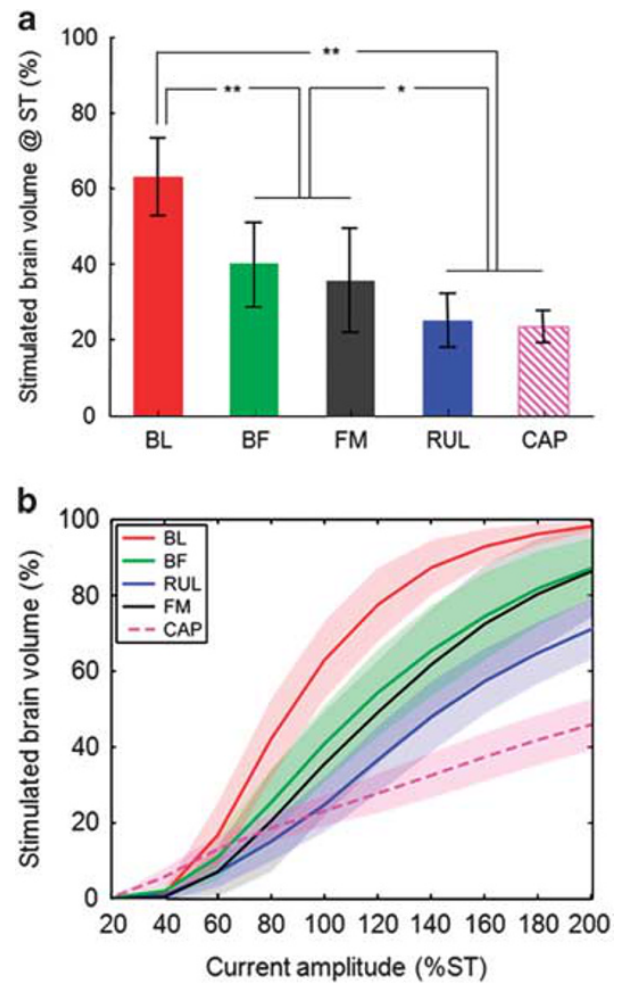

Figure 4 Percentage brain volume stimulated above the neural activation threshold $\left(E \geqslant E_{t h}\right)(a)$ at the measured individual $S T$ and $(b)$ as a function of current amplitude relative to the individual ST for $\mathrm{BL}, \mathrm{BF}, \mathrm{FM}$, and RUL ECT as well as CAP MST. In panel (a), the bars and error bars correspond, respectively, to the mean and SD of the individual averages of the four subjects. In panel (b), the lines and shaded regions correspond, respectively, to the mean and SD of the individual averages of the four subjects. Note that the values at the 100\% ST point on the $x$ axis in plot (b) are the same as the values in plot (a). ${ }^{*}{ }^{*} p<0.0001, * p<0.02$.

variability owing to interindividual differences, Figure 5 compares the coefficient of variation of the stimulated brain volume among the four subjects for current amplitude equal to the individual ST vs current amplitude that is fixed. The latter is set to the average ST across subjects within modality. For all modalities, individualized current amplitude results in less variability of the stimulated brain volume (16-36\%) 


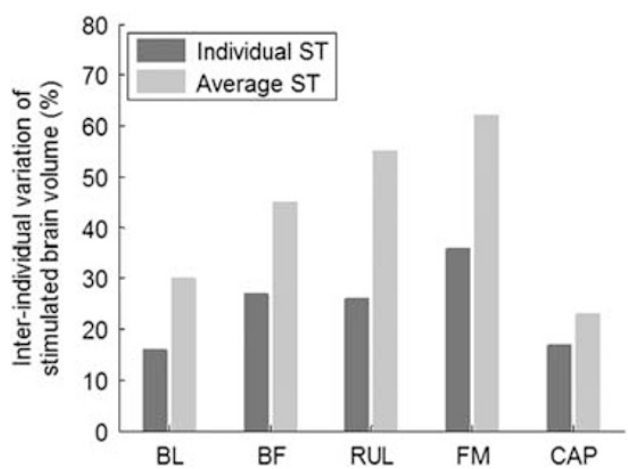

Figure 5 Effect of modality and current amplitude individualization on interindividual differences of stimulated brain subvolume. The coefficient of variation of the stimulated brain subvolume across the four NHP subjects is compared between the cases when the current amplitude is set to the individual ST (dark gray) vs a non-individualized fixed value equal to average ST within modality (gray).

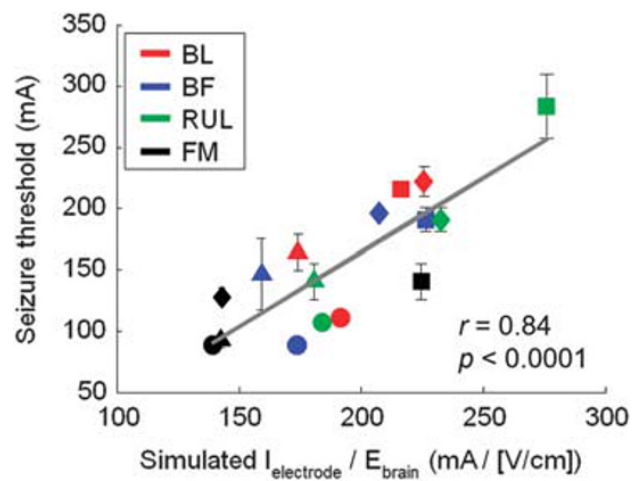

Figure 6 Correlation between the average individual amplitude-titrated ST and the $/ / E_{\text {brain }}$ ratio computed from individual E-field simulation models for the four ECT modalities. Each marker color represents a different ECT modality, as indicated by the legend. Each marker shape represents an NHP subject, (circle $=M A$, square $=C H$, diamond $=D Y$, and triangle $=R Z$ ). The separate correlations for each ECT modality as well as for each MST are shown in Supplementary Figure SI.

compared with fixed current amplitude (23-62\%). The difference between the coefficients of variation for individualized $v s$ fixed current amplitude is smallest for MST, suggesting that individualizing the current amplitude is more salient in ECT than in MST.

\section{Model Prediction of Amplitude-Titrated ST}

As the modality- and subject-specific E-field models capture individual anatomical variability, they could be used potentially to estimate the selection of the stimulus current amplitude for dose individualization. Figure 6 shows the relationship between the amplitude-titrated ST across the four ECT modalities and $I / E_{\text {brain }}$ computed from the corresponding individual E-field models. $I / E_{\text {brain }}$ is the electrode current amplitude required to generate 90th percentile E-field strength of $1 \mathrm{~V} / \mathrm{cm}$ in the brain. Figure 6 demonstrates a strong positive correlation $(r=0.84$, $p<0.0001$ ), suggesting that $I / E_{\text {brain }}$ is indeed a good predictor of ST. Similarly, the regression analysis of the
$Z$-scores of $I / E_{\text {brain }}$ and ST across all modalities, including MST, confirmed the predictive value of $I / E_{\text {brain }}\left(\mathrm{F}_{1}=93.0\right.$, $p<0.0001$; correlations for each individual modality are shown in Supplementary Figure S1). ST was not significantly influenced by the stimulation session, consistent with prior findings (Peterchev et al, 2015).

\section{DISCUSSION}

\section{Increased Stimulation Focality with Reduced Current Amplitude}

This study provides the first quantitative estimate of the spatial distribution of stimulation in the primate brain when ECT and MST are applied at the lowest current amplitude required to induce a seizure. The five modalities in this study differ in the strength of stimulation of various brain regions such as primary motor cortex, DLPFC, and hippocampus, as well as in the total brain subvolume exposed to suprathreshold E-field. Whereas direct comparison of the amplitudetitrated STs is not possible between NHPs and humans owing to the different head size and anatomy (Peterchev et al, 2015), the stimulation exposure of ROIs and the brain subvolume that needs to be stimulated to induce a seizure with a particular electrode or coil configuration are more likely to be comparable across the two species. For ECT, the stimulated subvolume at the amplitude-titrated ST in this study-ranging from $63 \%$ for $\mathrm{BL}$ to $25 \%$ for RUL-is substantially lower than the estimated 94-99\% for conventional electrode placements and current amplitude in humans (Lee et al, 2016). Figure 4b suggests that, to achieve widespread stimulation of the brain matching conventional ECT, the current amplitude has to be increased by more than twofold relative to the amplitude-titrated ST. Conversely, by applying the results of this study to a model of the stimulation strength and focality of human ECT (Lee et al, 2016), the amplitude-titrated ST in humans can be estimated to be in the $250-350 \mathrm{~mA}$ range for conventional electrode placements (BL, RUL, and BF) and approximately $420 \mathrm{~mA}$ for FM. These values are consistent with clinical studies showing that generalized seizures can be induced with the lowest current of conventional ECT devices (500 mA) (Mayur et al, 2015; Rosa et al, 2011; Rosa et al, 2012; Youssef and Sidhom, 2014) and with even lower currents when titrated by amplitude (Nahas et al, 2013; Peterchev et al, 2010).

For MST with a circular coil on vertex, the focality with amplitude titration in the NHPs (23\%) is comparable to the estimated focality at the device maximum amplitude in humans (21\%) (Lee et al, 2016). Thus it appears that the current amplitude used in clinical MST may be close to the lowest level required for seizure induction. Also, the MST focality in this study is comparable to that of amplitudetitrated RUL ECT (25\%), consistent with modeling studies in humans (Lee et al, 2016). However, even though the size of the brain subvolume stimulated above threshold is well matched, RUL ECT and CAP MST affect brain regions such as primary motor cortex and hippocampus to a different extent owing to the shape and placement of the respective electrodes and coil. Nevertheless, we showed previously in simulations that the spatial characteristics of the ECT E-field can be matched very well to MST by appropriate 
configuration of the electrodes and stimulus current amplitude; the pulse shape of ECT can be easily matched to MST as well (Deng, 2013; Deng et al, 2013a). Although at stimulation intensities near the neural activation threshold there are qualitative differences in the neural populations recruited by transcranial magnetic $v s$ electric stimulation, at or above the amplitude-titrated ST these differences are likely insignificant owing to the large extent of markedly suprathreshold neuronal stimulation and the comparable pulse width $(\sim 0.2 \mathrm{~ms}$ for both ECT and the dominant middle phase of the MST pulse) (Deng et al, 2011). Thus this study contributes to a body of evidence that ECT with low current amplitude and focal electrode placement can induce seizures with E-field that is focal and targeted similarly to MST. This is significant as ECT devices require less power, do not have coil heating issues, and are smaller and less expensive than MST devices.

\section{Reduction of Stimulation Variability with Current Amplitude Individualization}

Figure 5 indicates that, compared with fixed ECT current amplitude, individualization of the amplitude results in a reduction of the variation in stimulated brain subvolume by $40-53 \%$ across subjects. On the other hand, this improvement is only $26 \%$ for MST. This observation is consistent with our prior experimental and simulation studies of ECT and MST, suggesting that fixed current amplitude results in variable E-field strength and focality among subjects owing to interindividual differences in head size and anatomy, more so for ECT than for MST (Deng et al, 2015; Lisanby et al, 2003; Peterchev et al, 2015), and that amplitude individualization could reduce this variability (Deng et al, 2013a; Lee et al, 2015). Notably, the subjects in this study were all male, which likely reduces interindividual variability as females may differ from males in head anatomy and ST (Deng et al, 2015). The larger variability if females are included may strengthen the case for current amplitude individualization. Collectively, these findings suggest that current amplitude individualization could be a meaningful approach to compensate for interindividual variability in anatomy and neurophysiological excitability, which appears more significant in ECT than in MST. This could, in turn, reduce outcome variability of these interventions.

There are several approaches to individualize the current amplitude. First, ST can be titrated in the current amplitude domain (Liberson, 1948; Nahas et al, 2013). By virtue of the lower current amplitudes applied during amplitude-domain titration, it is expected that the effects of the titration procedure on the ST estimate are smaller than for conventional titration with fixed, high amplitude stimuli. Second, the MT can be used instead owing to its strong correlation with the amplitude-titrated ST (Peterchev et al, 2015). Third, MRI-based computer simulation can be used to account for the individual anatomical variability, as evaluated experimentally for the first time in this study. In the latter approach, the simulated electrode or coil current per E-field strength in the brain, $I / E_{\text {brain, }}$ captures the effect of individual anatomical variability. The $I / E_{\text {brain }}$ metric exhibited a strong positive correlation with the measured ST when all modalities were pooled, although it was significant only for RUL ECT when considered separately for each modality, which is unsurprising as only four subjects were studied. This correlation suggests that anatomical variability, captured by the individual models, may be an important factor driving individual differences in ST and thus dosing requirements for ECT and MST. Overall, these findings lend support, albeit limited, to the use of individual E-field models as a predictor of amplitude-titrated ST and hence as a means to individualize the stimulus current amplitude without the need to induce a seizure.

\section{Implications for ECT and MST Dosing}

This study does not provide information on the clinical efficacy and side effects of the characterized ECT and MST modalities. However, the quantitative information generated in this study could be useful for trials to evaluate targeting of specific brain regions with various strengths (controlled by the electrode/coil configuration and current amplitude relative to the amplitude-titrated ST) and other parameters (pulse width, frequency, number of pulses). For example, the parameters of stimulation could be manipulated to expose specific ROIs to intense E-field both below and above amplitude-titrated ST to assess the contribution of the E-field $v s$ the seizure. Further, amplitude titration to maximally focalize ECT could be carried out not only with ultrabrief but also with brief $(\geqslant 0.5 \mathrm{~ms})$ pulses, allowing decoupling of the effects of focality and pulse width. This is of interest as brief pulse ECT not only appears to have more robust therapeutic effects but also more side effects than ultrabrief-pulse modalities (Cretaz et al, 2015; Rasmussen, 2015). Also, the number of pulses and their frequency can be manipulated while keeping the current low and therefore focalizing the E-field stimulation. This is of interest as increasing the number of pulses can enhance the efficacy of relatively focal paradigms such as RUL ECT while keeping side effects low (Loo et al, 2014; Sackeim et al, 2000; Semkovska et al, 2016). Finally, the current amplitude individualization could be important for reducing outcome variability across subjects also for paradigms using currents higher than the amplitudetitrated ST.

\section{Limitations}

The head and brain anatomy of NHPs differs from that of humans. For example, the prefrontal cortex forms $28.5 \%$ of the neocortex in the human brain but only $11.3 \%$ in the macaque brain (Passingham, 2009). Therefore, results on the proportion of stimulated brain volume and ROI E-field exposure should be considered approximate in the context of human ECT and MST. We characterized only a limited set of ECT electrode placements and a single circular MST coil placement. Some MST studies use a double-cone coil instead (Kayser et al, 2015; Sun et al, 2016), which we have simulated previously (Deng et al, 2011, 2013b). Further, the accuracy of simulations of the E-field generated by electric or magnetic stimulation is limited by uncertainties in the geometry, electrical properties, and relative placement of the head tissues and the stimulating electrodes or coil. Nevertheless, in our modeling approach the calibration of the E-field solution to an empirical neural activation threshold could compensate for some modeling errors, as the E-field used to estimate the neural activation threshold at MT and the E-field at the 
amplitude-titrated ST are both affected in the same way. The assumed single neural activation threshold is obviously approximate but is reasonable given the similar neuronal composition of cortex across the brain and the relatively strong E-fields generated by ECT and MST. The small sample size is a further limitation; therefore results concerning individual variation should be interpreted with caution.

\section{CONCLUSIONS}

Coupling experimental threshold titrations with computer modeling in NHPs, this study quantified for the first time the spatial extent of brain stimulation necessary to induce a seizure with ECT and MST. Estimating the E-field induced in the brain allows direct comparison with human studies, which is confounded if solely the stimulus current amplitudes are considered. At the amplitude-titrated ST, which represents the individual minimum stimulus current amplitude required for seizure induction, the portion of the brain stimulated above the neural activation threshold is significantly less than for conventional ECT with high fixed current. This demonstrated quantitatively that ECT paradigms with lower than standard current amplitudes can induce seizures with more focal stimulation. The brain subvolume as well as specific brain regions stimulated at ST depends on the ECT electrode and MST coil configuration. For ECT, the BL and RUL electrode placements are least and most focal, respectively. Current-amplitude-titrated RUL ECT matches the focality of CAP MST, although they differ in the strength of stimulation in specific brain regions. Coupled with the results of previous simulation studies, this work shows that by manipulating the electrode or coil configuration and the current amplitude, seizures can be induced with relatively selective stimulation with either MST or ECT, which is technologically simpler. In addition to determining the most focal stimulus current, individual current amplitude titration reduces the interindividual variability in the E-field exposure of the brain. Finally, this work provided preliminary validation of prior modeling studies showing that individual E-field models could inform individualization of the current amplitude without the need for ST titration. Collectively, these insights could contribute toward the development of rational dosing strategies for seizure therapies with improved risk/benefit ratio.

\section{FUNDING AND DISCLOSURE}

This work was supported by the National Institutes of Health under grant R01MH091083 and an ANSYS Strategic Partnership. SH Lisanby has served as Principal Investigator or co-investigator on industry-sponsored research grants to Duke (Brainsway, NeoSync, Nexstim); equipment loans to Duke (Magstim, MagVenture); is co-inventor on a patent and patent applications on TMS/MST technology; has been supported by grants from NIH (R01MH091083, U01MH084241, R01MH060884), Stanley Medical Research Institute, US Air Force, and Brain and Behavior Foundation; and has no consultancies, speakers bureau memberships, board affiliations, or equity holdings in related industries. AV Peterchev is inventor on patents and patent applications on TMS/MST technology; has received research and travel support as well as patent royalties from Rogue Research for TMS technology, research and travel support as well as equipment loan from Tal Medical related to TMS technology, patent application support from Magstim, as well as TMS/MST equipment loans from MagVenture. The other authors declare no conflict of interest.

\section{ACKNOWLEDGMENTS}

We thank Dr Paolo Maccarini and Dr Murali Kadiramangalam for the ANSYS license donation. Preliminary results from this study were presented in part in conference proceedings (Lee et al, 2012b, 2013).

\section{REFERENCES}

Cretaz E, Brunoni AR, Lafer B (2015). Magnetic seizure therapy for unipolar and bipolar depression: a systematic review. Neural Plast 2015: 521398.

Deng Z-D (2013). Electromagnetic field modeling of transcranial electric and magnetic stimulation: targeting, individualization, and safety of convulsive and subconvulsive applications. $\mathrm{PhD}$ thesis, Columbia University, New York, NY, USA.

Deng ZD, Lisanby SH, Peterchev AV (2011). Electric field strength and focality in electroconvulsive therapy and magnetic seizure therapy: a finite element simulation study. J Neural Eng 8: 016007.

Deng ZD, Lisanby SH, Peterchev AV (2013a). Controlling stimulation strength and focality in electroconvulsive therapy via current amplitude and electrode size and spacing: comparison with magnetic seizure therapy. J ECT 29: 325-335.

Deng ZD, Lisanby SH, Peterchev AV (2013b). Electric field depthfocality tradeoff in transcranial magnetic stimulation: simulation comparison of 50 coil designs. Brain Stimul 6: 1-13.

Deng ZD, Lisanby SH, Peterchev AV (2015). Effect of anatomical variability on electric field characteristics of electroconvulsive therapy and magnetic seizure therapy: a parametric modeling study. IEEE Trans Neural Syst Rehabil Eng 23: 22-31.

Kayser S, Bewernick BH, Matusch A, Hurlemann R, Soehle M, Schlaepfer TE (2015). Magnetic seizure therapy in treatmentresistant depression: clinical, neuropsychological and metabolic effects. Psychol Med 45: 1073-1092.

Kellner CH, Fink M, Knapp R, Petrides G, Husain M, Rummans T et al (2005). Relief of expressed suicidal intent by ECT: a consortium for research in ECT study. Am J Psychiatry 162: 977-982.

Kellner CH, Knapp R, Husain MM, Rasmussen K, Sampson S, Cullum $M$ et al (2010). Bifrontal, bitemporal and right unilateral electrode placement in ECT: randomised trial. Br J Psychiatry 196: 226-234.

Lee WH, Deng ZD, Kim TS, Laine AF, Lisanby SH, Peterchev AV (2012a). Regional electric field induced by electroconvulsive therapy in a realistic finite element head model: influence of white matter anisotropic conductivity. Neuroimage 59: 2110-2123.

Lee WH, Lisanby SH, Laine AF, Peterchev AV (2012b). Stimulation strength and focality of electroconvulsive therapy with individualized current amplitude: a preclinical study. Conf Proc IEEE Eng Med Biol Soc 2012: 6430-6433.

Lee WH, Lisanby SH, Laine AF, Peterchev AV (2013). Electric field characteristics of electroconvulsive therapy with individualized current amplitude: a preclinical study. Conf Proc IEEE Eng Med Biol Soc 2013: 3082-3085.

Lee WH, Lisanby SH, Laine AF, Peterchev AV (2015). Electric field model of transcranial electric stimulation in nonhuman primates: correspondence to individual motor threshold. IEEE Trans Biomed Eng 62: 2095-2105. 
Lee WH, Lisanby SH, Laine AF, Peterchev AV (2016). Comparison of electric field strength and spatial distribution of electroconvulsive therapy and magnetic seizure therapy in a realistic human head model. Eur Psychiatry 36: 55-64.

Liberson WT (1948). Brief stimulus therapy; psysiological and clinical observations. Am J Psychiatry 105: 28-39.

Lisanby SH, Moscrip T, Morales O, Luber B, Schroeder C, Sackeim HA (2003). Neurophysiological characterization of magnetic seizure therapy (MST) in non-human primates. Suppl Clin Neurophysiol 56: 81-99.

Loo CK, Katalinic N, Smith DJ, Ingram A, Dowling N, Martin D et al (2014). A randomized controlled trial of brief and ultrabrief pulse right unilateral electroconvulsive therapy. Int J Neuropsychopharmacol 18: 1-8.

Mayur P, Harris A, Gangadhar B (2015). 500-mA ECT-a proof of concept report. J ECT 31: e23-e26.

Nahas Z, Short B, Burns C, Archer M, Schmidt M, Prudic J et al (2013). A feasibility study of a new method for electrically producing seizures in man: focal electrically administered seizure therapy [FEAST]. Brain Stimul 6: 403-408.

Passingham R (2009). How good is the macaque monkey model of the human brain? Curr Opin Neurobiol 19: 6-11.

Paxinos G, Huang XF, Toga AW (2000). The Rhesus Monkey Brain in Stereotaxic Coordinates. Academic Press: San Diego, CA, USA.

Peterchev AV, Krystal AD, Rosa MA, Lisanby SH (2015). Individualized low-amplitude seizure therapy: minimizing current for electroconvulsive therapy and magnetic seizure therapy. Neuropsychopharmacology 40: 2076-2084.

Peterchev AV, Rosa MA, Deng ZD, Prudic J, Lisanby SH (2010). Electroconvulsive therapy stimulus parameters: rethinking dosage. J ECT 26: 159-174.

Pons JP, Segonne E, Boissonnat JD, Rineau L, Yvinec M, Keriven R (2007). High-quality consistent meshing of multi-label datasets. Inf Process Med Imaging 20: 198-210.

Rasmussen KG (2015). Improving ECT efficacy and decreasing cognitive side effects. In: Reti IM (ed). Brain Stimulation: Methodologies and Interventions. Wiley Blackwell: Hoboken, NJ, USA, pp 83-106.

Reti IM (2015). How does electroconvulsive therapy work? In: Reti IM(ed). Brain Stimulation: Methodologies and Interventions. Wiley Blackwell: Hoboken, NJ, USA, pp 107-122.
Rosa MA, Abdo GL, Lisanby SH, Peterchev AV (2011). Seizure induction with low-amplitude-current $(0.5 \mathrm{~A})$ electroconvulsive therapy. J ECT 27: 341-342.

Rosa MA, Abdo GL, Rosa MO, Lisanby SH, Peterchev AV (2012). Fronto-medial electrode placement with low current amplitude: a case report. J ECT 28: 146.

Rossini PM, Barker AT, Berardelli A, Caramia MD, Caruso G, Cracco RQ et al (1994). Non-invasive electrical and magnetic stimulation of the brain, spinal cord and roots: basic principles and procedures for routine clinical application. Report of an IFCN committee. Electroencephalogr Clin Neurophysiol 91: 79-92.

Sackeim HA (2004). Convulsant and anticonvulsant properties of electroconvulsive therapy: towards a focal form of brain stimulation. Clin Neurosci Res 4: 39-57.

Sackeim HA, Prudic J, Devanand DP, Nobler MS, Lisanby SH, Peyser S et al (2000). A prospective, randomized, double-blind comparison of bilateral and right unilateral electroconvulsive therapy at different stimulus intensities. Arch Gen Psychiat 57: 425-434.

Saleem KS, Logothetis NK (2007). A Combined MRI and Histology Atlas of the Rhesus Monkey Brain in Stereotaxic Coordinates, 1st edn. Elsevier/Academic Press: Amsterdam, The Netherlands; Boston, MA, USA, 336-pp.

Semkovska M, Landau S, Dunne R, Kolshus E, Kavanagh A, Jelovac A et al (2016). Bitemporal versus high-dose unilateral twice-weekly electroconvulsive therapy for depression (EFFECT-Dep): a pragmatic, randomized, non-inferiority trial. Am J Psychiatry 173: 408-417.

Sun Y, Farzan F, Mulsant BH, Rajji TK, Fitzgerald PB, Barr MS et al (2016). Indicators for remission of suicidal ideation following magnetic seizure therapy in patients with treatment-resistant depression. JAMA Psychiatry 73: 337-345.

Tor PC, Bautovich A, Wang MJ, Martin D, Harvey SB, Loo C (2015). A systematic review and meta-analysis of brief versus ultrabrief right unilateral electroconvulsive therapy for depression. J Clin Psychiatry 76: e1092-e1098.

Weiner RD (2015). Introduction to convulsive therapy. In: Reti IM (ed). Brain Stimulation: Methodologies and Interventions. Wiley Blackwell: Hoboken, NJ, USA, pp 61-82.

Youssef NA, Sidhom E (2014). Examination of cognitive profile and variability in the current amplitude domain of low current amplitude ECT. Biol Psychiatry 75: 259s-260s.

Supplementary Information accompanies the paper on the Neuropsychopharmacology website (http://www.nature.com/npp) 